\title{
Anle138b and related compounds are aggregation specific fluorescence markers and reveal high affinity binding to $\alpha$-synuclein aggregates
}

\author{
Andreas A. Deeg ${ }^{\mathrm{a}, 1}$, Anne M. Reiner ${ }^{\mathrm{a}, 1}$, Felix Schmidt ${ }^{\mathrm{b}}$, Florian Schueder ${ }^{\mathrm{a}}$, Sergey Ryazanov ${ }^{\mathrm{c}}$, Viktoria C. Ruf ${ }^{\mathrm{b}}$, \\ Karin Giller ${ }^{c}$, Stefan Becker ${ }^{c}$, Andrei Leonov ${ }^{c}$, Christian Griesinger ${ }^{c, d}$, Armin Giese ${ }^{b}$, Wolfgang Zinth ${ }^{\text {a,* }}$ \\ a BioMolekulare Optik and Center for Integrated Protein Science CIPSM, Ludwig-Maximilians-Universität München, Oettingenstr. 67, 80538 Munich, Germany \\ b Zentrum für Neuropathologie und Prionforschung, Ludwig-Maximilians-Universität München, Feodor-Lynen-Str. 23, 81377 Munich, Germany \\ c NMR-based Structural Biology, Max Planck Institute for Biophysical Chemistry, Am Fassberg 11, 37077 Göttingen, Germany \\ ${ }^{\mathrm{d}}$ DFG Research Center Nanoscale Microscopy and Molecular Physiology of the Brain (CNMPB), Göttingen, Germany
}

\section{A R T I C L E I N F O}

\section{Article history:}

Received 26 January 2015

Received in revised form 26 April 2015

Accepted 27 May 2015

Available online 29 May 2015

\section{Keywords:}

Protein aggregation

Amyloid dye

Anle138b

$\alpha$-Synuclein

Fluorescence marker

Parkinson's disease

\begin{abstract}
A B S T R A C T
Background: Special diphenyl-pyrazole compounds and in particular anle138b were found to reduce the progression of prion and Parkinson's disease in animal models. The therapeutic impact of these compounds was attributed to the modulation of $\alpha$-synuclein and prion-protein aggregation related to these diseases.

Methods: Photophysical and photochemical properties of the diphenyl-pyrazole compounds anle138b, anle186b and sery $313 \mathrm{~b}$ and their interaction with monomeric and aggregated $\alpha$-synuclein were studied by fluorescence techniques.

Results: The fluorescence emission of diphenyl-pyrazole is strongly increased upon incubation with $\alpha$-synuclein fibrils, while no change in fluorescence emission is found when brought in contact with monomeric $\alpha$-synuclein. This points to a distinct interaction between diphenyl-pyrazole and the fibrillar structure with a high binding affinity $\left(K_{d}=190 \pm 120 \mathrm{nM}\right)$ for anle138b. Several $\alpha$-synuclein proteins form a hydrophobic binding pocket for the diphenyl-pyrazole compound. A UV-induced dehalogenation reaction was observed for anle138b which is modulated by the hydrophobic environment of the fibrils.

Conclusion: Fluorescence of the investigated diphenyl-pyrazole compounds strongly increases upon binding to fibrillar $\alpha$-synuclein structures. Binding at high affinity occurs to hydrophobic pockets in the fibrils.

General significance: The observed particular fluorescence properties of the diphenyl-pyrazole molecules open new possibilities for the investigation of the mode of action of these compounds in neurodegenerative diseases. The high binding affinity to aggregates and the strong increase in fluorescence upon binding make the compounds promising fluorescence markers for the analysis of aggregation-dependent epitopes.
\end{abstract}

(c) 2015 Elsevier B.V. All rights reserved

\section{Introduction}

In several neurodegenerative disorders like Parkinson's and prion diseases the abnormal aggregation of proteins into highly ordered structures so called amyloids can be observed [1,2]. These aggregates, located in the brain of infected individuals, are characterized by a cross- $\beta$-sheet protein structure and a fibrillar morphology under the electron microscope. The amyloid aggregates are considered to play an important role in the pathogenesis of neurodegenerative diseases [3-7]. Furthermore, recent experiments pointed out that not only the fibrillar structures but especially the formation of smaller, highly cytotoxic oligomeric forms [8] might lead to neuronal loss [9-12]. At present, the mechanism by which protein aggregation to fibrillar and oligomeric

\footnotetext{
* Corresponding author.

E-mail address: wolfgang.zinth@physik.uni-muenchen.de (W. Zinth).

1 Andreas A. Deeg and Anne M. Reiner contributed equally and should be considered shared first authors.
}

structures leads to dysfunction of neurons and neuronal loss is not really known.

A promising progress for the development of new pharmaceutical agents and for a better understanding of the origin of neurodegenerative diseases is the investigation of small organic compounds that modulate the disease related protein aggregation [1,13-15]. Based on this approach diphenyl-pyrazole (DPP) compounds, in particular anle138b, were identified recently as new pharmaceutical agents for the treatment of prion and Parkinson's diseases, where the aggregation of prion and $\alpha$-synuclein proteins plays an important role [16-18]. The potential of the DPP compounds to modulate prion protein aggregation was first discovered in high throughput fluorescence correlation screenings by Scanning for Intensely Fluorescent Targets (SIFT) $[13,19,20]$ and in prion cell culture assays $[17,20,21]$. After extensive medicinal chemistry optimization using SIFT and the prion cell culture assay, further SIFT experiments also showed the potential of the compounds to inhibit $\alpha$-synuclein aggregation. The most promising molecules were tested in various mouse models of prion and Parkinson's diseases. Pronounced 
prolongation of the survival time and the disease free survival respectively could be observed for several DPP-compounds, in particular for anle138b $[16,17,20]$. Examination of brain extracts revealed a reduction of aggregated species of the prion and $\alpha$-synuclein proteins $[16,18]$. These observations suggested that in vivo the mode of action of the DPP-compounds is based on the modulation of protein aggregation [16]. However, the molecular details of aggregation modulation and the interaction of the DPP-compounds with monomeric or aggregated forms of proteins remain to be elucidated.

In this paper we investigate by fluorescence spectroscopy the interaction of $\alpha$-synuclein as monomers and fibrils with different potential pharmaceutical agents of the 3,5-diphenyl-pyrazole (DPP) type. These compounds bind to fibrillar structures and show a strongly increased fluorescence emission upon binding, which could be used for targeting and diagnostic purposes. We determine binding parameters and investigate the photoreactions of the therapeutically most efficient compound anle138b.

\section{Materials and methods}

\subsection{Fluorescence measurements}

All fluorescence measurements were carried out in fused silica cuvettes (Hellma $10 \times 4 \mathrm{~mm}$, Typ $117.04 \mathrm{~F}-\mathrm{QS}$ ) with a fluorescence spectrometer (Horiba Fluorolog3), equipped with an anisotropy module (FL-1044). The excitation wavelength was set to $300 \mathrm{~nm}$, where all investigated compounds show a pronounced absorption band. To establish the homogeneity of the solutions a home-built steering system was used for all measurements. The emission spectra and titration data for the determination of binding parameters were recorded in front-face geometry (anisotropy measurements in right angle geometry) with a low excitation power of $2 \mu \mathrm{W}$ (excitation monochromator settings: $0.4 \mathrm{~nm}$ bandwidth, emission monochromator settings: $8 \mathrm{~nm}$ bandwidth). For these settings the precision of the fluorescence measurements is limited. However with this low excitation power, light induced modifications of the samples can be avoided. To increase the precision of the emission spectra of the compounds the weak fluorescence of the corresponding solvent or protein solution was subtracted for all measurements. For the investigation of the compounds in different solvents $50 \mu \mathrm{M}$ and $25 \mu \mathrm{M}$ solutions of the molecules in DMSO (Uvasol, Merck) were prepared. These solutions were diluted 1:100 ( $\mathrm{v} / \mathrm{v}$ as always used in the following text) to obtain a $500 \mathrm{nM}$ concentration in toluene (Uvasol, Merck) and glycerin $/ \mathrm{H}_{2} \mathrm{O}$ (Sigma, LiChrosolv) $(80: 20)$ or a $250 \mathrm{nM}$ concentration of the compounds in buffer ( $50 \mathrm{mM}$ Tris- $\mathrm{HCl}, \mathrm{pH}$ 7). The measurements were performed at these low concentrations since the solubility of the compounds in aqueous solutions is limited to the sub-micromolar regime (see SI). At such a small solubility the high sensitivity of fluorescence spectroscopy is required to perform a detailed analysis of the interaction of the compounds with target proteins.

For fluorescence and anisotropy measurements on anle138b with monomeric and aggregated proteins the compound was first dissolved to $10 \mathrm{mM}$ in DMSO and then directly diluted to $250 \mathrm{nM}$ in buffer ( $50 \mathrm{mM}$ Tris- $\mathrm{HCl}, \mathrm{pH} 7$ ). $800 \mu$ of this $250 \mathrm{nM}$ anle138b solution with $0.0025 \%$ DMSO was filled in two cuvettes (cuvette 1 and cuvette 2). Monomeric $(50 \mu \mathrm{M})$ and fibrillar (about $13 \mu \mathrm{M}$ protein concentration) $\alpha$-synuclein solutions were added to cuvettes 1 and 2 respectively to yield a concentration of about $4 \mu \mathrm{M} \alpha$-synuclein in each cuvette. The same amount of proteins was added to identical reference cuvettes (cuvette 3 and cuvette 4 ) containing only $800 \mu$ l buffer and fluorescence and anisotropy measurements were performed. The small emission from the reference cuvettes 3 and 4 was subtracted from the corresponding emission of cuvettes 1 and 2 . The measurements of sery313b and anle186b in combination with monomeric and fibrillar $\alpha$-synuclein were performed with an equivalent procedure.
For quantitative analysis of the binding of anle $138 \mathrm{~b}$ to $\alpha$ synuclein fibrils three different solutions of $10 \mu \mathrm{M}, 25 \mu \mathrm{M}$ and $50 \mu \mathrm{M}$ anle138b in DMSO were prepared. These solutions were diluted directly into three cuvettes containing buffer ( $50 \mathrm{mM}$ Tris- $\mathrm{HCl}$, $\mathrm{pH} 7$ ) to yield an anle138b-concentration of $100 \mathrm{nM}, 250 \mathrm{nM}$ and $500 \mathrm{nM}$ respectively and $1 \%$ DMSO. In addition a reference cuvette was prepared containing only buffer and 1\% DMSO. Subsequently the same amount of a fibrillar stock solution was added to all cuvettes and the fluorescence was recorded at $345 \mathrm{~nm}$. The fluorescence of the reference cuvette was subtracted. This experiment was performed with a fibrillar stock solution of $P_{s}=52 \mu \mathrm{M}$ protein concentration and $P_{S}=21 \mu \mathrm{M}$ in an independent control experiment (see SI).

For the investigation of the photoreactions of anle $138 \mathrm{~b}$, the $300 \mathrm{~nm}$ light of the spectrofluorometer was used for fluorescence excitation and also for the illumination of the compounds. For this purpose the settings of the spectrofluorometer were modified: All measurements were performed in right angle geometry with an excitation power of $30 \mu \mathrm{W}$ (excitation monochromator settings: $0.8 \mathrm{~nm}$ bandwidth, emission monochromator settings: $2 \mathrm{~nm}$ bandwidth). Three cuvettes (cuvettes 5, 6 and 7) containing $800 \mu \mathrm{l}$ of $250 \mathrm{nM}$ anle138b in buffer ( $50 \mathrm{mM}$ Tris- $\mathrm{HCl}$, pH 7) with $0.0025 \%$ DMSO were prepared as mentioned above. Small amounts of stock solutions containing $\alpha$-synuclein monomer $(50 \mu \mathrm{M})$ or fibrils (about $24 \mu \mathrm{M}$ protein concentration) were added to cuvettes 5 and 6 to yield a concentration of $6.8 \mu \mathrm{M}$ and $3.2 \mu \mathrm{M} \alpha$-synuclein respectively. The three cuvettes were illuminated with $300 \mathrm{~nm}(30 \mu \mathrm{W})$ for $45 \mathrm{~min}$. During illumination the fluorescence at $345 \mathrm{~nm}$ was measured every $22 \mathrm{~s}$. Corresponding reference cuvettes with buffer (cuvette 8 ) and with the equal amount of $\alpha$-synuclein monomer in buffer with $0.0025 \%$ DMSO (cuvette 9 ) and fibrils in buffer with $0.0025 \%$ DMSO (cuvette 10 ) were measured under identical conditions. These reference measurements were subtracted from the corresponding data of the measurements with anle138b.

The time resolved fluorescence measurements were performed with a Hamamatsu streak camera (C5680-24 C) and a femtosecond laseramplifier-system (Clark CPA 2001, $180 \mathrm{fs}, 778$ nm, $1 \mathrm{kHz}$ ). For the excitation of the compound $300 \mathrm{~nm}$ light pulses (pulse energy $40 \mathrm{~nJ}$ ) were generated by a noncollinear optical parametric amplifier. The compound sery313b was first dissolved in DMSO $(50 \mu \mathrm{M})$ and diluted to $500 \mathrm{nM}$ in water for the time resolved measurement (see details in the supporting information).

\subsection{Absorption measurements}

All absorption measurements were performed in fused silica cuvettes (Hellma $10 \times 4 \mathrm{~mm}$, Typ 117.04F-QS, optical path length $4 \mathrm{~mm}$ ) with a Shimadzu UV 1800 absorption spectrometer. The compounds anle138b, anle186b and sery313b were dissolved in ethanol (Uvasol, $\mathrm{c}=10 \mu \mathrm{M}$ ) and stirred during the measurements. To avoid the formation of photoproducts, low illumination intensities were chosen.

The concentrations of the $\alpha$-synuclein proteins in the fibrillar solutions were determined via measurements of the tyrosine absorption (extinction coefficient at $280 \mathrm{~nm} 1209 \mathrm{M}^{-1} \mathrm{~cm}^{-1}$ ) [22].

\subsection{Data analysis}

For the quantitative analysis of the dissociation constant $K_{d}$ and the binding stoichiometry i.e., the number $B$ of proteins in the aggregate required to form one binding site, the fluorescence amplitude $F\left(A_{0}, P\right)$ was recorded as a function of the total concentrations of anle138b $\left(A_{0}\right)$ and $\alpha$-synuclein $(P)$. In the analysis it is assumed that only anle138b molecules bound to protein lead to a detectable fluorescence signal, while the emission of unbound anle138b is negligible (see below for a justification). Thus the fluorescence amplitude is proportional to the 
concentration $A_{b}$ of bound anle138b, $F\left(A_{0}, P\right)=F=y \cdot A_{b}$. The concentrations were analyzed according to the law of mass action.

$K_{d}=\frac{P_{u} \cdot A_{u}}{A_{b}}=\frac{\left(P / B-A_{b}\right) \cdot\left(A_{0}-A_{b}\right)}{A_{b}}$

$A_{u}$ describes the concentration of the unbound anle138b molecules. $P_{u}$ is the concentration of the free binding sites in the solution, which can be expressed by the total $\alpha$-synuclein concentration $P$, the number $B$, and the concentration $A_{b}$ of the bound compound. The addition of the aggregated proteins to the cuvette reduces the concentration $A_{u}$ of unbound anle $138 \mathrm{~b}$ and increases the fluorescence. In the experiment the actual concentration of anle $138 \mathrm{~b} A_{0}$ is determined by the initial anle $138 \mathrm{~b}$ concentration in the solution $a_{s}$, and the dilution by the addition of the solution containing the aggregated $\alpha$-synuclein. For a protein concentration $P_{s}$ of the stock solution of aggregated proteins and the actual protein concentration $P$ in the cuvette one obtains: $A_{0}=a_{s} \cdot\left(1-P / P_{s}\right)$. Elimination of $A_{0}$ and $A_{b}$ in Eq. (1) leads to:

$K_{d}=\frac{(P / B-F / y) \cdot\left(\left(1-P / P_{S}\right) a_{s}-F / y\right)}{F / y}$.

The measurement of the fluorescence $F$ for different $\alpha$-synuclein concentrations $P$ and different values $a_{s}$ yields a set of experimental data. A fit of these experimental data to Eq. (2) allows the determination of the parameters $K_{d}, B$ and $y$ using a global fitting procedure (Mathematica Wolfram, NonlinearModelFit). Due to the small solubility of anle138b in aqueous solutions, to adhesion effects and to the limited accuracy of the dilution process, there are uncertainties in the values of the concentration $a_{s}$ which are considered in the fitting procedure (see SI).

The reaction quantum yield for the photoreaction of anle138b was calculated from the light induced fluorescence increase (see Fig. 4) as described in the supporting information.

\subsection{Protein preparation}

\subsubsection{Expression and purification of recombinant wildtype $\alpha$-synuclein}

Expression and purification were performed as described previously [23]. Briefly, pET-5a/ $\alpha$-synuclein (136TAT) plasmid (wt-plasmid by Philipp Kahle, LMU Munich; 136-TAC/TAT-Mutation by Matthias Habeck) was used to transform Escherichia coli BL21(DE3)pLys (Novagen, Madison, WI, USA), and expression was induced with isopropyl ß-D-1-thiogalactopyranoside (IPTG, Peqlab, Erlangen, Germany). Cells were lysed by boiling after heat-inactivation of proteases. After centrifugation the supernatant was filtered through a Filtropur S 0.2 filter (Sarstedt, Nümbrecht, Germany), loaded on a HiTrap Q HP anionexchange column ( $5 \mathrm{ml}$, GE Healthcare, Munich, Germany) and eluted with a linear $25 \mathrm{mM}$ to $500 \mathrm{mM} \mathrm{NaCl}$-gradient. The synuclein-fraction was gel filtrated via a Superdex 75 prep grade column $(25 \mathrm{ml}$, GE Healthcare, Munich, Germany) and protein concentration was assessed to $1 \mathrm{mg} / \mathrm{ml}$ in $50 \mathrm{mM}$ Tris- $\mathrm{HCl}$, $\mathrm{pH}$ 7. After freezing in liquid nitrogen the protein was stored at $-80^{\circ} \mathrm{C}$.

\subsubsection{Fibrillization of recombinant wildtype $\alpha$-synuclein}

As described previously, fibrillization was induced by agitation at high protein concentrations [24,25]. Briefly, $1 \mathrm{mg} / \mathrm{ml} \alpha$-synuclein in $50 \mathrm{mM}$ Tris- $\mathrm{HCl}$, containing $100 \mathrm{mM} \mathrm{NaCl}, 0.02 \% \mathrm{NaN}_{3}$, pH 7 was incubated for $96 \mathrm{~h}$ at $37^{\circ} \mathrm{C}$ and $1400 \mathrm{rmp}$ using an Eppendorf Thermomixer Comfort (Eppendorf, Hamburg, Germany). After freezing in liquid nitrogen fibrils were stored at $-80{ }^{\circ} \mathrm{C}$. In this preparation protocol $\alpha$ synuclein fibrils are formed, as validated by electron microscopy and ThT fluorescence (see SI).

\section{5. $L C-M S$}

Analytical and preparative high performance liquid chromatography (HPLC) was performed by using a Waters HPLC system with a Waters 996 Photodiode Array Detector. All separations involved a mobile phase of $0.1 \%$ trifluoroacetic acid (TFA) (v/v) in water (solvent $\mathrm{A}$ ) and $0.1 \%$ TFA in acetonitrile (solvent B). HPLC was performed by using a reversed-phase (RP) column Eurospher RP 18, $100 \AA$, $5 \mu \mathrm{m}, 250 \times$ $4.6 \mathrm{~mm}$ (analytical) and $250 \times 16 \mathrm{~mm}$ (preparative) at flow rates of $1 \mathrm{ml} \cdot \mathrm{min}^{-1}$ (analytical) and $7 \mathrm{ml} \cdot \mathrm{min}^{-1}$ (preparative). Electrospray ionization mass spectrometry (ESI-MS) and liquid chromatography/ mass spectrometry (LC/MS) analyses were obtained by using a Waters Micromass ZQ 4000 mass spectrometer in conjunction with the Waters HPLC apparatus described above.

\section{Results and discussion}

\subsection{Absorption and emission properties}

The photophysical properties and the interaction of 3,5-diphenylpyrazole (DPP) compounds with $\alpha$-synuclein protein are investigated in detail for the three compounds anle138b, sery313b and anle186b (see Scheme 1). The UV/Vis absorption spectra of the compounds (dissolved in ethanol) are given as insets in Fig. 1a, b, and c. The absorption spectra of the three compounds are similar. They depend weakly on the solvent properties (see SI). The absorption spectra show a pronounced band at about $270 \mathrm{~nm}$ and a shoulder around $300 \mathrm{~nm}$. Here the molar extinction coefficient $\varepsilon$ is in the order of $\varepsilon \sim 10,000 \mathrm{l} /(\mathrm{cm} \mathrm{mol})$. Strength and position of the observed absorption bands point to a delocalized electronic system. The fluorescence properties of the DPP-compounds in different solvents are shown in Figs. 1 and 2 for excitation in the long-wavelength absorption band at $300 \mathrm{~nm}$. Considering potential photochemical reactions of the DPP-compounds (see below for a detailed description) we used very weak illumination powers which lead to a limited precision of the fluorescence measurements. The curves of Fig. 1a, b and c show the fluorescence spectra of anle138b, sery313b and anle186b in a glycerol-water mixture (red, $500 \mathrm{nM}$ ) and in toluene (blue, $500 \mathrm{nM})$. The spectra of the compounds ( $250 \mathrm{nM})$ in aqueous buffer (black) are scaled by a factor of two, to allow a direct comparison to the spectra in toluene and glycerol/water. In aqueous buffer the fluorescence quantum yield $(\mathrm{QY})$ of anle $138 \mathrm{~b}$ is very small $(\mathrm{QY}<0.5 \%)$ and no significant fluorescence can be detected with the measurement settings used. The emission of sery $313 \mathrm{~b}$ and anle $186 \mathrm{~b}$ is stronger and readily detected. The spectra in aqueous buffer extend from $330 \mathrm{~nm}$ to over $500 \mathrm{~nm}$ with two broad peaks around $340 \mathrm{~nm}$ and $420 \mathrm{~nm}$. The fluorescence quantum yields (QYs) are considerably larger than that of anle138b. We find values of $Q Y=(17.3 \pm 6.6) \%$ for sery $313 \mathrm{~b}$ and $\mathrm{QY}=(8.6 \pm 6.6) \%$ for anle186b. When anle138b is dissolved in solvents, which are less polar than water or have a higher viscosity, one finds larger amplitudes of the fluorescence emission and a changed emission spectrum. For toluene (which has the smallest polarity, dielectric constant $\mathrm{DC}=2.39$ and a viscosity $\eta=0.59 \mathrm{mPa} \mathrm{s}$ [26]), we find the peak around $335 \mathrm{~nm}$. In the more viscous glycerol-water mixture ( $D C \approx 50.6$ and $\eta \approx 45.9 \mathrm{mPa}$ s at $25^{\circ} \mathrm{C}[27,28]$ ) the peak is red-shifted by ca. $7 \mathrm{~nm}$ and an extended red wing of the fluorescence appears. Similar spectral changes are observed for the other DPP-compounds sery313b and anle186b (see Fig. 1b and c). Most significant is the strong change in the fluorescence emission when these compounds are dissolved in polar solvent water. Here a red wing emission, poorly visible in the less polar solvents, dominates the fluorescence.

The strong solvent dependence of the fluorescence emission of the pharmaceutically active DPP-compounds implies that fluorescence can be used to monitor the interaction with $\alpha$-synuclein. In a first step, the DPP-compounds were investigated in combination with monomeric $\alpha$-synuclein. The addition of different amounts of monomeric proteins to the DPP-compounds in aqueous buffer did not lead to a significant 
<smiles>Brc1cccc(-c2cc(-c3ccc4c(c3)OCO4)n[nH]2)c1</smiles><smiles>Fc1cccc(-c2cc(-c3ccc4c(c3)OCO4)n[nH]2)c1</smiles><smiles>Oc1cccc(-c2cc(-c3ccc4c(c3)OCO4)n[nH]2)c1</smiles>

Scheme 1. Structures of the compounds anle138b, anle186b and sery313b.

change of the fluorescence properties (see Fig. 1, right). These observations give no indication for a specific interaction or binding between monomeric $\alpha$-synuclein and the DPP-compounds. The situation is different for fibrils. As shown in Fig. $1 \mathrm{~d}$ to $\mathrm{f}$, the addition of fibrils is leading to a change in the fluorescence spectrum and to a strong increase in the fluorescence intensity around $335 \mathrm{~nm}$ for all DPP-compounds. For sery313b and anle $186 \mathrm{~b}$ the fluorescence increase upon binding occurs around $335 \mathrm{~nm}$ and is correlated with the decrease of the red wing fluorescence at $\lambda>385 \mathrm{~nm}$. The strong changes in the fluorescence properties of the DPP-compounds support the idea of a specific binding to fibrillar structures at a location well shielded from the aqueous solution. This is evident from the comparison of the fluorescence spectra from anle138b in aqueous solution containing $\alpha$-synuclein fibrils and the spectrum obtained in the nonpolar solvent toluene. Both samples exhibit the same position of the emission peak (Fig. 2b) while a shift is seen for anle138b in the more polar mixture of glycerol/water (see Fig. 2a). Qualitatively, the same behavior could be observed for sery313b and anle186b (see SI).

A rise in fluorescence quantum yield of small aromatic molecules upon binding to amyloid fibrils is well known from molecules like thioflavin or N-arylaminonaphtalene sulfonates [29-31]. For these molecules the increase in fluorescence is assumed to originate from the rigid and/or hydrophobic environment at the binding site of the aggregates [30-32]. Although these compounds have been studied extensively, the fluorescence mechanism and the origin of the fluorescence increase upon binding are still controversial [31,33-35]. For the DPPcompounds one may assume that a rigid environment at the binding sites of the fibrillar aggregates [30] could reduce the intrinsic flexibility of the compounds and with that could slow down internal conversion processes. An alternative explanation would refer to the changes of polarity in the binding pocket. This has been shown recently for indigo compounds where hydrogen bonds and a hydrogen transfer strongly accelerated internal conversion [36]. In addition, photochemical reactions (see below) may reduce the lifetime of the fluorescent state. All these processes can change the lifetime $\tau$ of the excited DPPmolecules and would explain the observed strong increased fluorescence intensities upon binding to the fibrils.

Further information on binding of the DPP-compounds to the fibrils is obtained from studies of the fluorescence anisotropy. The fluorescence anisotropy $r$ (see Table 1) recorded in polarized emission experiments may contain information on the rotational correlation time $\theta$ of the molecules [37].

$$
r=\frac{r_{0}}{1+\tau / \theta}
$$
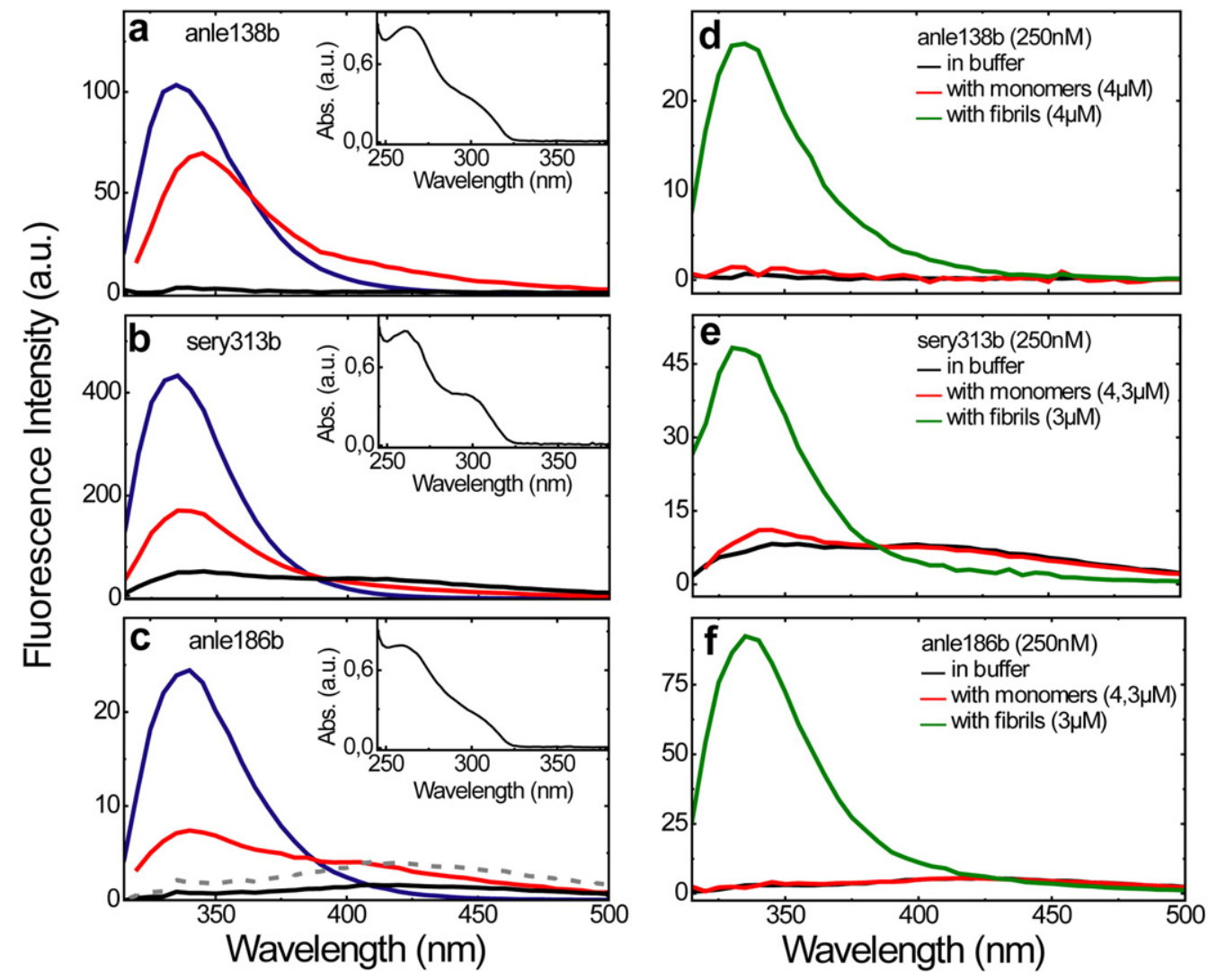

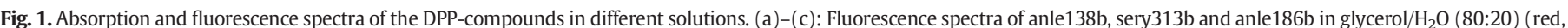

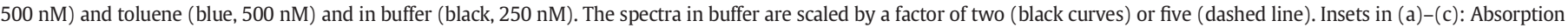

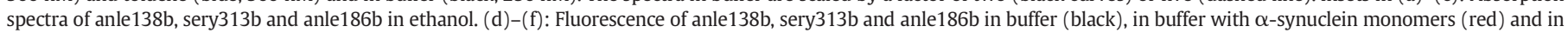
buffer with fibrils (green). 


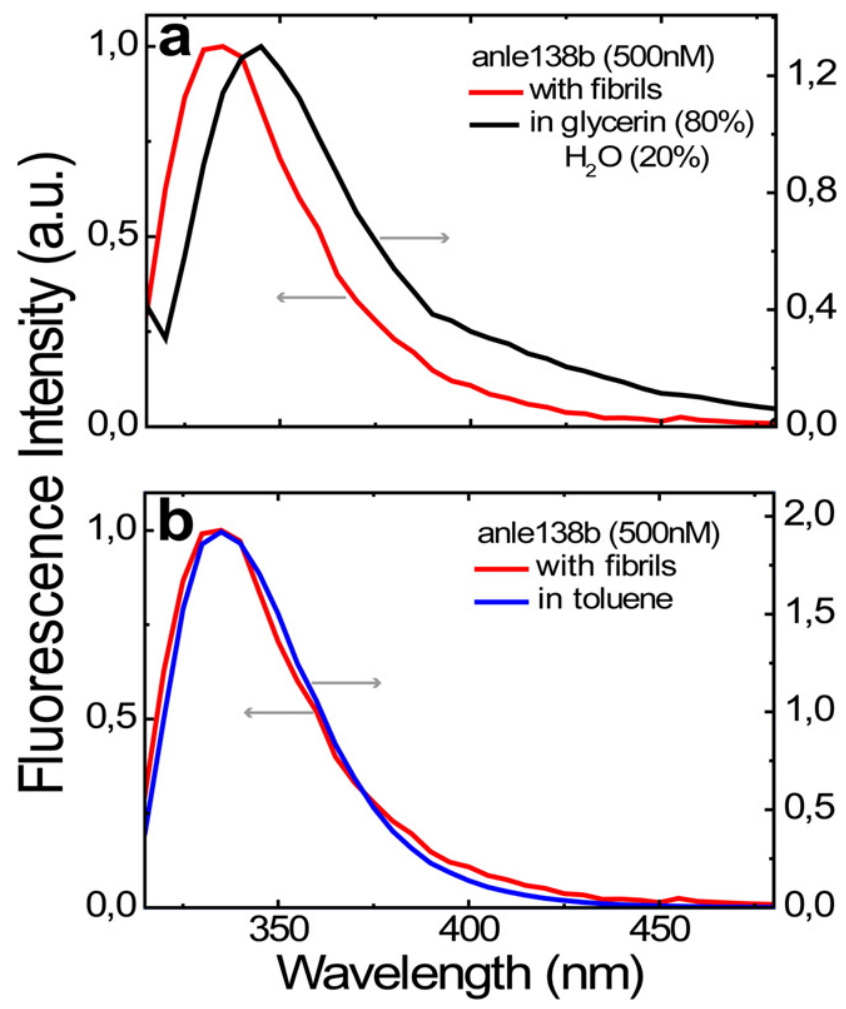

Fig. 2. Fluorescence spectra of anle138b in different solvents. (a) and (b) red spectra: Fluorescence of anle138b in buffer containing fibrils. (a) Black curve: Spectra of anle138b in glycerin/ $\mathrm{H}_{2} \mathrm{O}$ (80:20). (b) Blue curve: Spectra of anle138b in toluene. The emission intensity of anle138b with fibrils is normalized (see left axis labels). The relative fluorescence intensity of the spectra in toluene and glycerin/ $\mathrm{H}_{2} \mathrm{O}$ is indicated by the right axis labels.

Here $r_{0}$ is the intrinsic anisotropy of the transition for a slowly reorienting molecule with $\theta \gg \tau$. For sery313b we could determine the fluorescence lifetime $\tau$ from a streak-camera experiment, where a single exponential fit yielded a time constant $\tau$ of about $4 \mathrm{~ns}$. The vanishing fluorescence anisotropy of this compound in aqueous solution fits well to the short rotational diffusion time $\theta \approx 150$ ps expected for molecules with the size of the used DPP compounds [37]. For the $\alpha-$ synuclein monomer and fibrils the orientational motion is much slower and in the order of $\theta=1-2$ ns and $\theta \gg 10$ ns respectively [38]. When sery $313 \mathrm{~b}$ is rigidly bound to the protein large values of $r$ are expected. Indeed we find for sery $313 \mathrm{~b}$ combined with fibrillar $\alpha$-synuclein that the fluorescence anisotropy rises to a value of $r=0.2$. This large value points to a strong reduction of the rotational diffusion of sery $313 \mathrm{~b}$ and to strong interactions with the fibrils. At the same time, the negligible anisotropy of sery $313 \mathrm{~b}$ in solutions with monomeric $\alpha$-synuclein indicates that sery313b does not bind rigidly to the monomers.

For the other DPP-compounds a similar behavior is found. Table 1 shows the fluorescence anisotropies of the investigated DPPcompounds. The measured anisotropies in solutions without proteins and with monomeric $\alpha$-synuclein are very low. However, the combination with fibrillar $\alpha$-synuclein leads to strong anisotropies of $r=0.2-$ 0.3. These observations indicate that the compounds anle138b, anle $186 \mathrm{~b}$ and sery $313 \mathrm{~b}$ are rigidly bound to the $\alpha$-synuclein fibrils.

Table 1

Fluorescence anisotropy at $345 \mathrm{~nm}$ of anle138b, sery313b and anle186b in buffer, in buffer with $\alpha$-synuclein monomer and in buffer with $\alpha$-synuclein fibrils.

\begin{tabular}{llrr}
\hline Anisotropy at 345 nm & Anle138b & \multicolumn{1}{c}{ Sery313b } & \multicolumn{1}{c}{ Anle186b } \\
\hline In buffer & - & $0.006 \pm 0.02$ & $0.02 \pm 0.02$ \\
Buffer + monomers & - & $-0.002 \pm 0.06$ & $0.003 \pm 0.1$ \\
Buffer + fibrils & $0.3 \pm 0.06$ & $0.2 \pm 0.08$ & $0.3 \pm 0.1$ \\
\hline
\end{tabular}

\subsection{Quantitative binding experiments with anle $138 b$}

In the pharmaceutical studies in mouse models anle138b has proven to be one of the most potent molecules for the treatment of neurodegenerative diseases. In this regard the interaction and in particular the dissociation constant $K_{d}$ and the stoichiometry of the binding of anle $138 \mathrm{~b}$ to aggregated structures of $\alpha$-synuclein are of high interest. In general, these parameters are determined by titration experiments, where the binding ligand (in our case anle138b) is titrated to a solution with a defined concentration of receptor molecules [30,39]. Due to the small solubility of anle138b in aqueous solution this approach cannot be performed. In order to obtain quantitative information on the dissociation constant $K_{d}$ and the stoichiometry of the binding process, concentration dependent fluorescence measurements were performed where the concentrations of compounds and protein were varied. Fig. 3 shows titration experiments where the fluorescence of anle138b at $345 \mathrm{~nm}$ is recorded for solutions with different concentrations of anle138b (100 nM, $250 \mathrm{nM}, 500 \mathrm{nM}$ ). The concentration of the fibrillar stock solution was $P_{s}=52 \mu \mathrm{M}$ in this experiment. The fluorescence intensities of anle138b rise upon addition of $\alpha$-synuclein fibrils, due to the interaction of the DPP-compound with the fibrillar aggregates. For high protein concentrations each curve saturates when all anle138b molecules are bound to the aggregated structures. This titration experiment was repeated in an independent control measurement with a fibrillar stock solution of $P_{S}=21 \mu \mathrm{M}$.

The two titration measurements were modeled independently by Eq. (2) in a global fit procedure as described in Materials and methods [40]. From this modeling the dissociation constant $K_{d}=190 \pm$ $120 \mathrm{nM}$ and the number $B$ of $\alpha$-synuclein units required to form one binding site in the fibrils can be obtained, $B=7.2 \pm 2.2$ (for details see SI). Apparently several $\alpha$-synuclein units combined in the particular structure of the fibrillar aggregates are required to form one binding pocket. The dissociation constant in the $200 \mathrm{nM}$ range and the number of $\alpha$-synuclein monomers forming one binding site are small compared to results from investigations of thioflavin $T$. For the binding of thioflavin $\mathrm{T}$ to fibrils formed of the protein $\mathrm{A} \beta$ values of $K_{d}=750 \mathrm{nM}$ and $B=40.5$ were determined [41]. The strong binding of anle138b to aggregated $\alpha$-synuclein supports the key role of the interactions with misfolded $\alpha$-synuclein in the therapeutic action.

Misfolded protein aggregates are considered to be a key element in the pathogenesis of neurodegenerative diseases, since they were

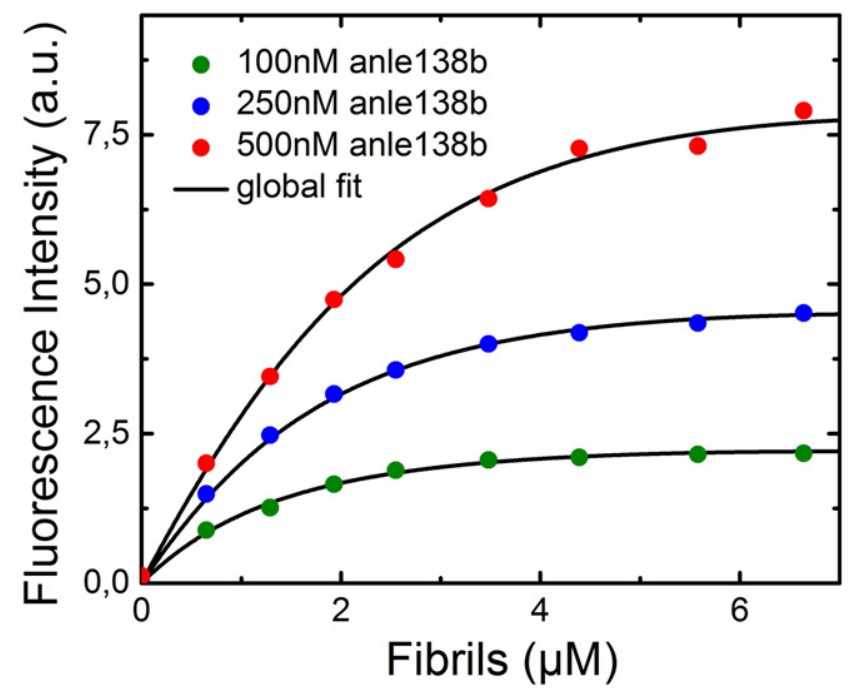

Fig. 3. Fluorescence intensity at $345 \mathrm{~nm}$ measured as a function of the concentration of anle138b and the concentration of aggregated $\alpha$-synuclein fibrils (points). The black lines represent a global fit to the data according to Eq. (2) with variable $a_{s}$. This leads to deviations in $a_{s}$ from the nominal initial anle138b concentrations of $100 \mathrm{nM}, 250 \mathrm{nM}$ and $500 \mathrm{nM}$ to the fitted values $119 \mathrm{nM}, 247 \mathrm{nM}$ and $433 \mathrm{nM}$ respectively. 
found to be cytotoxic [3-6] or to induce the dysfunction of neurons and neuronal loss [7]. The binding of anle138b might inhibit aggregate growth and/or could reduce the toxicity of the aggregates and hamper disease progression as observed in in vivo experiments [16]. As small aggregates (oligomers) seem to be the most relevant toxic species in vivo, it is important to note that anle138b strongly reduced the amount of oligomers formed in vitro and in vivo [16]. However, whereas binding studies with fibrils can be done in a straightforward way, binding studies with oligomers are problematic as no protocols for the preparation of stable structures of high concentration oligomers are available and as various different oligomer species which differ in size and structure exist [42]. In preliminary experiments, we attempted to investigate the interaction of anle138b with a specific type of in-vitro generated $\alpha$-synuclein oligomers [42,43], and did not observe a change in fluorescence representative for rigid binding. This could be due to e.g., a missing interaction of anle138b to this specific type of oligomer [43, 44 ] or due to no significant change of the fluorescence properties of anle138b upon binding, or a rapid disintegration of the oligomeric structure upon binding the DPP-compound. Studies with more elaborate techniques are required to treat this important subject. Nevertheless the observed inhibition of oligomer formation in vitro [16,45] provides strong support for an important role of direct interactions of anle138b with oligomeric species as a relevant mechanism of action.

\subsection{Photoreaction of anle $138 b$}

It is well known in fluorescence spectroscopy, especially for halogen-benzene compounds that extended UV-illumination could induce photoreactions [46]. Therefore we kept the illumination during the fluorescence experiments on a very low level $(\sim 228 \mathrm{~nJ} / \mathrm{ml})$, where potential photoreactions do not influence the fluorescence measurement itself. In this paragraph we investigate the stability and the photochemical properties of the compounds in more detail.

The aqueous solutions containing DPP-compounds were illuminated with light at $300 \mathrm{~nm}$ and the samples were analyzed by LC-MS (see LCMS, supporting information). For the compound sery313b no photoreaction with accumulation of photoproducts could be observed even for strong illumination (absorbed dose $\sim 0.4 \mathrm{~mJ} / \mathrm{ml}$ ). In the case of anle186b illumination, LC-MS analysis demonstrated a slow decomposition of anle186b, but no distinct photoproduct could be identified. However, under almost the same illumination conditions (absorbed dose $\sim 0.6 \mathrm{~mJ} / \mathrm{ml}$ ) anle138b in water underwent a photo-induced dehalogenation reaction. LC-MS analysis of the products revealed that the bromine is substituted by an OH-group (see LC-MS, supporting information). In other words, anle138b is predominantly converted to sery $313 \mathrm{~b}$, but at the same time the general decomposition seen for anle186b is also observed for anle138b. The photochemical reactions of anle138b were also investigated in DMSO (at an absorbed dose of $\sim 60 \mathrm{~mJ} / \mathrm{ml}$ ). In this solvent anle138b is also dehalogenated and based on LC-MS analysis three main products have been identified (see SI-Fig. 7). Apparently the UV-induced cleavage of the C-Br bond presumably leads to the formation of a radical, which further reacts with the compounds present in the mixture.

It should be noted that the observed behavior suggests using anle138b as a photo-crosslinking agent. E.g., when anle138b is bound to the $\alpha$-synuclein fibrils photo-induced reactions with the peptide, with side chains or backbone, could allow obtaining structural information, for instance the location of the binding pocket.

For a more detailed analysis of the photoreaction of anle138b in aqueous buffer we studied the fluorescence of samples containing originally anle138b. We make use of the strong increase in fluorescence when anle138b is photo-converted (dehalogenated) to sery $313 \mathrm{~b}$, which has much higher fluorescence yield (see Fig. 1). In Fig. 4 we plotted the emission as a function of the dose absorbed by the sample prior to recording. Each point represents the fluorescence induced by an absorbed illumination dose at $300 \mathrm{~nm}$ of $5 \mu \mathrm{J} / \mathrm{ml}$. Fig. 4, blue squares,

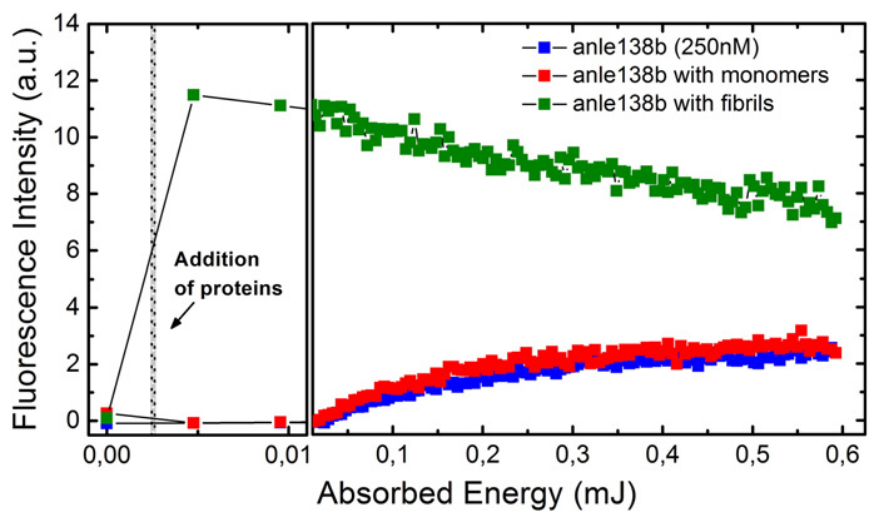

Fig. 4. Illumination dependent fluorescence of anle138b in buffer (blue squares), in buffer with $\alpha$-synuclein monomers (red squares) and in buffer with $\alpha$-synuclein fibrils (green squares). Monomers and fibrils were added at the gray line.

shows the fluorescence of anle138b in aqueous buffer recorded at $345 \mathrm{~nm}$. After a negligible emission with very low pre-illumination the fluorescence rises to the value known from sery313b. The reaction quantum yield $\Phi_{P C}$ of the photo-conversion can be calculated from the initial rise of the fluorescence intensity with the absorbed illumination dose (see Materials and methods). The reaction quantum yield $\Phi_{P C}$ is defined as the ratio of absorbed photons to the number of formed photochemical products (here sery313b). An unusually high value of $\Phi_{P C}=$ $400 \%$ is found. This high value indicates a radical chain reaction where one absorbed photon leads to the conversion of ca. four molecules anle138b to sery313b [47]. The light induced reaction was also studied by fluorescence spectroscopy of anle138b dissolved in DMSO (see SI) where different products have been observed. In these measurements the reaction (dehalogenation) quantum yield is much lower and a value of $\Phi_{P C}=30 \%$ is found. When anle $138 \mathrm{~b}$ is illuminated in a solution containing monomeric $\alpha$-synuclein at a concentration of $6.8 \mu \mathrm{M}$ (Fig. 4 , red squares) we observe exactly the same rise in fluorescence as in the protein-free aqueous solution. Apparently anle138b is surrounded by water molecules and no indication for a distinct interaction with $\alpha$ synuclein is found. Interesting features appear when anle138b is brought in contact with fibrils. In the experiment shown in Fig. 4 (green squares) fluorescence at $345 \mathrm{~nm}$ of anle138b in buffer solution is detected before and after the addition of $3.2 \mu \mathrm{M}$ fibrils. The first (reference) point before the gray line was recorded prior to the addition of the fibrils and shows very weak emission. A strong increase in fluorescence intensity is observed upon addition of fibrils. Apparently, as mentioned above, the change in surroundings leads to a strong increase in fluorescence yield. The subsequent exposure of the solution (anle138b with fibrils) to $300 \mathrm{~nm}$ light leads to a reduction of the fluorescence intensity (see Fig. 4, green squares). Obviously also for the anle138b molecule bound to fibrils a photoconversion occurs. The photoproduct has a smaller fluorescence than the original fibril-bound anle138b. The slope of the change in emission indicates that this photo-induced conversion is less efficient, than in the case of a fibril-free aqueous environment. This observation again indicates that the anle138b molecules, in the binding pocket of the fibrils, are shielded from the water environment.

\section{Conclusion}

In this paper the photophysical and photochemical properties of the three DPP-compounds anle138b, anle186b and sery313b and their interaction with solvents and with monomeric and aggregated $\alpha$ synuclein were studied by fluorescence techniques. Measurements of the DPP-compounds in different solvents show that the fluorescence strongly depends on the physical properties of the environment. For DPP-solutions containing monomeric $\alpha$-synuclein no change of the fluorescence properties and thus no indications for a specific interaction 
are found. In contrast, the DPP-compounds mixed with $\alpha$-synuclein fibrils show a strong increase in fluorescence intensity and anisotropy. The analysis of concentration dependent emission reveals that only a combination of several $(7.2 \pm 2.2) \alpha$-synuclein monomers in the fibrillar aggregate forms one binding site for anle138b. These binding sites show a high binding affinity with $K_{d}=190 \pm 120 \mathrm{nM}$ for anle138b. The fluorescence spectrum indicates that the binding site is hydrophobic. Here the bound anle138b is protected from the surrounding water molecules. Binding to the fibrils also modulates the photochemical dehalogenation reaction identified for anle138b. This observation again indicates that anle138b in the binding pocket is well shielded from water.

The missing interaction of the compounds to monomeric structures and the high binding affinity to fibrillar aggregates of the in vivo active DPP-compounds support the idea that the mode of action of these novel therapeutic agents might be related to the interaction with structural epitopes related to the formation of pathological aggregates. Furthermore, the compounds presented in this work, with their particular fluorescence properties, are promising candidates as fluorescence markers for the analysis of neurodegenerative diseases.

\section{Acknowledgments}

This work was supported by the Deutsche Forschungsgemeinschaft (Center for Integrated Protein Science Munich, CIPSM and SFB 749 A5), Lüneburg Foundation and the Max Planck Society (to C.G.). We thank Franziska F. Graupner for the time-resolved fluorescence measurements.

\section{Appendix A. Supplementary data}

Supplementary data to this article can be found online at http://dx. doi.org/10.1016/j.bbagen.2015.05.021.

\section{References}

[1] G.B. Irvine, O.M. El-Agnaf, G.M. Shankar, D.M. Walsh, Protein aggregation in the brain - the molecular basis for Alzheimer's and Parkinson's diseases, Mol. Med. 14 (2008) 451-464, http://dx.doi.org/10.2119/2007-00100.Irvine.

[2] S.B. Prusiner, Prions, Proc. Natl. Acad. Sci. U. S. A. 95 (1998) 13363-13383.

[3] V. Novitskaya, O.V. Bocharova, I. Bronstein, I.V. Baskakov, Amyloid fibrils of mammalian prion protein are highly toxic to cultured cells and primary neurons, J. Biol. Chem. 281 (2006) 13828-13836, http://dx.doi.org/10.1074/jbc.M511174200.

[4] M.F. Mossuto, et al., The non-core regions of human lysozyme amyloid fibrils influence cytotoxicity, J. Mol. Biol. 402 (2010) 783-796, http://dx.doi.org/10.1016/j.jmb. 2010.07.005.

[5] M. Bucciantini, et al., Toxic effects of amyloid fibrils on cell membranes: the importance of ganglioside GM1, FASEB J. 26 (2012) 818-831, http://dx.doi.org/10.1096/fj. 11-189381.

[6] A. Lorenzo, B.A. Yankner, Amyloid fibril toxicity in Alzheimer's disease and diabetes, Ann. N. Y. Acad. Sci. 777 (1996) 89-95.

[7] J. Tsai, J. Grutzendler, K. Duff, W.-B. Gan, Fibrillar amyloid deposition leads to local synaptic abnormalities and breakage of neuronal branches, Nat. Neurosci. 7 (2004) 1181-1183, http://dx.doi.org/10.1038/nn1335.

[8] G. Bitan, E.A. Fradinger, S.M. Spring, D.B. Teplow, Neurotoxic protein oligomers what you see is not always what you get, Amyloid 12 (2005) 88-95, http://dx.doi. org/10.1080/13506120500106958.

[9] M. Bucciantini, et al., Inherent toxicity of aggregates implies a common mechanism for protein misfolding diseases, Nature 416 (2002) 507-511, http://dx.doi.org/10. $1038 / 416507 a$

[10] M.D. Kirkitadze, G. Bitan, D.B. Teplow, Paradigm shifts in Alzheimer's disease and other neurodegenerative disorders: the emerging role of oligomeric assemblies, J. Neurosci. Res. 69 (2002) 567-577, http://dx.doi.org/10.1002/jnr.10328.

[11] B.S. Gadad, G.B. Britton, K.S. Rao, Targeting oligomers in neurodegenerative disorders: lessons from alpha-synuclein, tau, and amyloid-beta peptide, J. Alzheimers Dis. 24 (2011) 223-232, http://dx.doi.org/10.3233/JAD-2011-110182.

[12] D.P. Karpinar, et al., Pre-fibrillar alpha-synuclein variants with impaired betastructure increase neurotoxicity in Parkinson's disease models, EMBO J. 28 (2009) 3256-3268, http://dx.doi.org/10.1038/emboj.2009.257.

[13] U. Bertsch, et al., Systematic identification of antiprion drugs by high-throughput screening based on scanning for intensely fluorescent targets, J. Virol. 79 (2005) 7785-7791, http://dx.doi.org/10.1128/JVI.79.12.7785-7791.2005.

[14] N.W. Schiffer, et al., Identification of anti-prion compounds as efficient inhibitors of polyglutamine protein aggregation in a zebrafish model, J. Biol. Chem. 282 (2007) 9195-9203, http://dx.doi.org/10.1074/jbc.M607865200.

[15] J. Bieschke, et al., Small-molecule conversion of toxic oligomers to nontoxic betasheet-rich amyloid fibrils, Nat. Chem. Biol. 8 (2012) 93-101, http://dx.doi.org/10. 1038/nchembio.719.
[16] J. Wagner, et al., Anle138b: a novel oligomer modulator for disease-modifying therapy of neurodegenerative diseases such as prion and Parkinson's disease, Acta Neuropathol. 125 (2013) 795-813.

[17] F. Leidel, et al., Diphenylpyrazole-derived compounds increase survival time of mice after prion infection, Antimicrob. Agents Chemother. 55 (2011) 4774-4781, http:// dx.doi.org/10.1128/AAC.00151-11.

[18] J. Levin, et al., The oligomer modulator anle138b inhibits disease progression in a Parkinson mouse model even with treatment started after disease onset, Acta Neuropathol. 127 (2014) 779-780, http://dx.doi.org/10.1007/s00401-014-1265-3.

[19] J. Bieschke, et al., Ultrasensitive detection of pathological prion protein aggregates by dual-color scanning for intensely fluorescent targets, Proc. Natl. Acad. Sci. 97 (2000) 5468-5473, http://dx.doi.org/10.1073/pnas.97.10.5468.

[20] A. Giese, et al., New drugs for inhibiting aggregation of proteins involved in diseases linked to protein aggregation and/or, neurodegenerative diseases, WO/ 2010/0003722010.

[21] M. Geissen, et al., From high-throughput cell culture screening to mouse model: identification of new inhibitor classes against prion disease, ChemMedChem 6 (2011) 1928-1937, http://dx.doi.org/10.1002/cmdc.201100119.

[22] N. Errington, A.J. Doig, A phosphoserine-lysine salt bridge within an alpha-helical peptide, the strongest alpha-helix side-chain interaction measured to date, Biochemistry 44 (2005) 7553-7558, http://dx.doi.org/10.1021/bi050297j.

[23] B. Nuscher, et al., $\alpha$-Synuclein has a high affinity for packing defects in a bilayer membrane a thermodynamics study, J. Biol. Chem. 279 (2004) 21966-21975, http://dx.doi.org/10.1074/jbc.M401076200.

[24] K.A. Conway, J.D. Harper, P.T. Lansbury, Fibrils formed in vitro from alpha-synuclein and two mutant forms linked to Parkinson's disease are typical amyloid, Biochemistry 39 (2000) 2552-2563, http://dx.doi.org/10.1021/bi991447r.

[25] A.L. Fink, Misbehaving Proteins, Springer, New York 2006, pp. 265-285.

[26] M.A. Haidekker, T.P. Brady, D. Lichlyter, E.A. Theodorakis, Effects of solvent polarity and solvent viscosity on the fluorescent properties of molecular rotors and related probes, Bioorg. Chem. 33 (2005) 415-425, http://dx.doi.org/10.1016/j.bioorg.2005. 07.005 .

[27] M.L. Sheely, Glycerol viscosity tables, Ind. Eng. Chem. 24 (1932) 1060-1064, http:// dx.doi.org/10.1021/ie50273a022.

[28] G.P. Association, Physical Properties of Glycerine and its Solutions, Glycerine Producers' Association, 1963.

[29] H. Naiki, K. Higuchi, M. Hosokawa, T. Takeda, Fluorometric determination of amyloid fibrils in vitro using the fluorescent dye, thioflavin T1, Anal. Biochem. 177 (1989) 244-249.

[30] M.S. Celej, E.A. Jares-Erijman, T.M. Jovin, Fluorescent N-arylaminonaphthalene sulfonate probes for amyloid aggregation of $\alpha$-synuclein, Biophys. J. 94 (2008) 4867-4879, http://dx.doi.org/10.1529/biophysj.107.125211.

[31] A. Hawe, M. Sutter, W. Jiskoot, Extrinsic fluorescent dyes as tools for protein characterization, Pharm. Res. 25 (2008) 1487-1499, http://dx.doi.org/10.1007/s11095007-9516-9.

[32] L. Brand, J.R. Gohlke, Fluorescence probes for structure, Annu. Rev. Biochem. 41 (1972) 843-868, http://dx.doi.org/10.1146/annurev.bi.41.070172.004211.

[33] L.S. Wolfe, et al., Protein-induced photophysical changes to the amyloid indicator dye thioflavin T, Proc. Natl. Acad. Sci. U. S. A. 107 (2010) 16863-16868.

[34] R. Khurana, et al., Mechanism of thioflavin T binding to amyloid fibrils, J. Struct. Biol. 151 (2005) 229-238, http://dx.doi.org/10.1016/j.jsb.2005.06.006.

[35] V.I. Stsiapura, et al., Thioflavin T as a molecular rotor: fluorescent properties of thioflavin T in solvents with different viscosity, J. Phys. Chem. B 112 (2008) 15893-15902, http://dx.doi.org/10.1021/jp805822c.

[36] M. Dittmann, et al., Photostabilität von 4,4'-Dihydroxythioindigo: ein Mimetikum des Indigo, Angew. Chem. 126 (2014) 602-605, http://dx.doi.org/10.1002/ange. 201307016.

[37] J.R. Lakowicz, Principles of Fluorescence Spectroscopy, Springer, 2007.

[38] S. Thirunavukkuarasu, E.A. Jares-Erijman, T.M. Jovin, Multiparametric fluorescence detection of early stages in the amyloid protein aggregation of pyrene-labeled $\alpha$ synuclein, J. Mol. Biol. 378 (2008) 1064-1073, http://dx.doi.org/10.1016/j.jmb. 2008.03.034.

[39] Y. Liang, Applications of isothermal titration calorimetry in protein science, Acta Biochim. Biophys. Sin. 40 (2008) 565-576.

[40] A.L. Lehninger, D.L. Nelson, M.M. Cox, Principles of Biochemistry, 2nd edition Worth Publishers. Inc, 1993.

[41] A. Lockhart, et al., Evidence for the presence of three distinct binding sites for the thioflavin T class of Alzheimer's disease PET imaging agents on $\beta$-amyloid peptide fibrils, J. Biol. Chem. 280 (2005) 7677-7684, http://dx.doi.org/10.1074/jbc.M412056200.

[42] M. Kostka, et al., Single particle characterization of iron-induced pore-forming alpha-synuclein oligomers, J. Biol. Chem. 283 (2008) 10992-11003, http://dx.doi. org/10.1074/jbc.M709634200.

[43] H.-Y. Kim, M.-K. Cho, D. Riedel, C. Fernandez, M. Zweckstetter, Dissociation of amyloid fibrils of alpha synuclein in supercooled water, Angew. Chem. Int. Ed. 47 (2008) 5046-5048.

[44] H.-Y. Kim, et al., Structural properties of pore-forming oligomers of $\alpha$-synuclein, J Am. Chem. Soc. 131 (2009) 17482-17489, http://dx.doi.org/10.1021/ja9077599.

[45] F. Schmidt, et al., Single-channel electrophysiology reveals a distinct and uniform pore complex formed by alpha-synuclein oligomers in lipid membranes, PLoS One 7 (2012).

[46] S. Fery-Forgues, N. Paillous, Photodehalogenation and photodimerization of 2-(4halophenyl)benzoxazoles. Dependence of the mechanism on the nature of the halogen atom, J. Org. Chem. 51 (1986) 672-677, http://dx.doi.org/10.1021/jo00355a017.

[47] W.H. Brown, B.L. Iverson, E. Anslyn, C.S. Foote, Organic Chemistry 7th, Wadsworth Cengage Learning, 2013. 